\title{
On the structure constants of volume preserving diffeomorphism algebra
}

\author{
Matsuo Sato ${ }^{\mathrm{a}}$ \\ Department of Natural Science, Faculty of Education, Hirosaki University, Bunkyo-cho 1, Hirosaki, Aomori 036-8560, Japan
}

Received: 4 April 2014 / Accepted: 28 April 2014 / Published online: 20 May 2014

(C) The Author(s) 2014. This article is published with open access at Springerlink.com

\begin{abstract}
Regularizing a volume preserving diffeomorphism (VPD) is equivalent to a long standing problem, namely regularizing a Nambu-Poisson bracket. In this paper, as a first step toward regularizing VPD, we find general complete independent bases of VPD algebra. Especially, we find a complete independent basis that gives simple structure constants, where three area preserving diffeomorphism algebras are manifest. This implies that an algebra that regularizes a VPD algebra should include three $u(N)$ Lie algebras.
\end{abstract}

\section{Introduction}

The area preserving diffeomorphism (APD) algebra is regularized by the $u(N)$ Lie algebra. Actually, a large $N$ limit of structure constants of $u(N)$ Lie algebra in the 't Hooft basis reduces to those of the APD algebra defined on $T^{2}$ [1-4]. Because the APD algebra is generated by the Poisson bracket, it is regularized by the Lie bracket of the $u(N)$ Lie algebra. This structure induces the following: the Heisenberg picture of quantum mechanics reduces to the canonical formalism of classical mechanics in the classical limit. Another application is that one can show that BFSS matrix theory and the IIB matrix model contain the lightcone supermembrane and the type IIB superstring, respectively, by using this regularization [5-7].

On the other hand, regularizing the Nambu-Poisson bracket is a long standing problem ${ }^{1}$ [14-37]. As in the case of APD, the Nambu-Poisson bracket generates a volume preserving diffeomorphism (VPD) algebra. In this paper, as a first step toward regularizing the Nambu-Poisson bracket, we search for several independent bases of the VPD algebra and obtain simple structure constants.

\footnotetext{
${ }^{1}$ For example, if the problem is solved, one should be possible to show that a three algebra model of M-theory [8-13] contains the semilightcone supermembrane.

a e-mail: msato@cc.hirosaki-u.ac.jp
}

\section{General complete independent bases of VPD algebra}

VPD is a diffeomorphism $x^{i} \rightarrow y^{i}(x)(i=1,2,3)$ that satisfies $\operatorname{det} \partial_{i} y^{j}(x)=1$. Then the infinitesimal transformation $y^{i}(x)=x^{i}+\delta x^{i}(x)$ satisfies

$\partial_{i} \delta x^{i}(x)=0$

Also $\delta x^{i}(x)=\epsilon^{i j k} \partial_{j} f(x) \partial_{k} g(x)$ satisfy this equation. Transformations of a scalar field generated by these solutions are given by

$$
\begin{aligned}
\delta Z(x) & \equiv \delta x^{i}(x) \partial_{i} Z(x) \\
& =\epsilon^{i j k} \partial_{i} f(x) \partial_{j} g(x) \partial_{k} Z(x) \\
& =\{f(x), g(x), Z(x)\} .
\end{aligned}
$$

This implies that the Nambu-Poisson bracket generates VPD. The transformations

$\delta=\delta x^{i}(x) \partial_{i}=\epsilon^{i j k} \partial_{i} f(x) \partial_{j} g(x) \partial_{k}$

form the VPD algebra.

The APD is a two-dimensional analog of the VPD. The infinitesimal transformations

$\delta=\delta X^{I}(Y) \partial_{I}=\epsilon^{I J} \partial_{I} F(Y) \partial_{J}$

on $T^{2}(I, J=1,2)$, where

$\partial_{I} \delta X^{I}(X)=0$

are spanned by the generators

$\delta(A)=i \mathrm{e}^{i A Y} \epsilon^{I J} A_{I} \partial_{J}$,

which are obtained by substituting $F(Y)=\mathrm{e}^{i A Y}$ into (2.4).

On the other hand, a complete independent basis of VPD cannot be obtained by substituting $f(x)=\mathrm{e}^{i a x}$ and $g(x)=\mathrm{e}^{i b x}$ into (2.3) because $\delta x^{i}(x)$ is a local vector in three 
dimensions. We need to solve (2.1). In the case of APD, (2.6) are complete independent solutions of (2.5). On $T^{3}$, we make a Fourier transformation, $\delta x^{i}(x)=\sum_{a} v^{i}(a) \mathrm{e}^{i a x}$. Equation (2.1) implies

$a_{i} v^{i}(a)=0$.

An independent solution of (2.7) is given by

$\bar{v}_{1}=\left(-a_{2}, a_{1}, 0\right)$,

$\bar{v}_{2}=a \times \bar{v}_{1}=\left(-a_{1} a_{3},-a_{2} a_{3}, a_{1}^{2}+a_{2}^{2}\right)$,

for $a=\left(a_{1}, a_{2}, a_{3}\right)$ (except $\left.a_{1}=a_{2}=0\right)$, and

$\bar{v}_{1}^{\prime}=(1,0,0)$,

$\bar{v}_{2}^{\prime}=(0,1,0)$,

for $a=\left(0,0, a_{3}\right)$.

The corresponding VPD generators are given by

$$
\begin{aligned}
& S_{1}(a)=\mathrm{e}^{i a x} \bar{v}_{1}^{i} \partial_{i}=\mathrm{e}^{i a x}\left(-a_{2} \partial_{1}+a_{1} \partial_{2}\right), \\
& S_{2}(a)=\mathrm{e}^{i a x} \bar{v}_{2}^{i} \partial_{i}=\mathrm{e}^{i a x}\left(-a_{1} a_{3} \partial_{1}-a_{2} a_{3} \partial_{2}\right. \\
& \left.\quad+\left(a_{1}^{2}+a_{2}^{2}\right) \partial_{3}\right), \\
& S_{1}^{\prime}\left(0,0, a_{3}\right)=\mathrm{e}^{i a_{3} x^{3}} \bar{v}_{1}^{\prime} \partial_{i}=\mathrm{e}^{i a_{3} x^{3}} \partial_{1}, \\
& S_{2}^{\prime}\left(0,0, a_{3}\right)=\mathrm{e}^{i a_{3} x^{3}} \bar{v}_{2}^{\prime} \partial_{i}=\mathrm{e}^{i a_{3} x^{3}} \partial_{2},
\end{aligned}
$$

which form the VPD algebra

$$
\begin{aligned}
& {\left[S_{1}(a), S_{1}(b)\right]=i\left(a_{1} b_{2}-a_{2} b_{1}\right) S_{1}(a+b),} \\
& {\left[S_{2}(a), S_{2}(b)\right]=i \alpha S_{1}(a+b)+i \beta S_{2}(a+b),} \\
& {\left[S_{1}(a), S_{2}(b)\right]=i \gamma S_{1}(a+b)+i \delta S_{2}(a+b),} \\
& {\left[S_{1}(a), S_{1}^{\prime}\left(0,0, b_{3}\right)\right]=-i a_{1} S_{1}(a+b),} \\
& {\left[S_{2}(a), S_{1}^{\prime}\left(0,0, b_{3}\right)\right]=-i a_{2} b_{3} S_{1}(a+b)-i a_{1} S_{2}(a+b),} \\
& {\left[S_{1}(a), S_{2}^{\prime}\left(0,0, b_{3}\right)\right]=-i a_{2} S_{1}(a+b),} \\
& {\left[S_{2}(a), S_{2}^{\prime}\left(0,0, b_{3}\right)\right]=i a_{1} b_{3} S_{1}(a+b)-i a_{2} S_{2}(a+b),} \\
& {\left[S_{1}^{\prime}\left(0,0, a_{3}\right), S_{1}^{\prime}\left(0,0, b_{3}\right)\right]=\left[S_{2}^{\prime}\left(0,0, a_{3}\right), S_{2}^{\prime}\left(0,0, b_{3}\right)\right]} \\
& \quad=\left[S_{1}^{\prime}\left(0,0, a_{3}\right), S_{2}^{\prime}\left(0,0, b_{3}\right)\right]=0,
\end{aligned}
$$

where

$$
\begin{aligned}
\alpha= & \frac{1}{\left(a_{1}+b_{1}\right)^{2}+\left(a_{2}+b_{2}\right)^{2}}\left(a_{2} b_{1}-a_{1} b_{2}\right)\left(\left(a_{1}^{2}+a_{2}^{2}\right) b_{3}^{2}\right. \\
& \left.+\left(b_{1}^{2}+b_{2}^{2}\right) a_{3}^{2}-2 a_{3} b_{3}\left(a_{1} b_{1}+a_{2} b_{2}\right)\right), \\
\beta= & \frac{1}{\left(a_{1}+b_{1}\right)^{2}+\left(a_{2}+b_{2}\right)^{2}}\left(( a _ { 1 } ^ { 2 } + a _ { 2 } ^ { 2 } ) b _ { 3 } \left(\left(a_{1}+b_{1}\right) b_{1}\right.\right. \\
& \left.+\left(a_{2}+b_{2}\right) b_{2}\right) \\
& \left.-\left(b_{1}^{2}+b_{2}^{2}\right) a_{3}\left(\left(a_{1}+b_{1}\right) a_{1}+\left(a_{2}+b_{2}\right) a_{2}\right)\right), \\
\gamma= & \frac{1}{a_{1}+b_{1}}\left(\frac { 1 } { ( a _ { 1 } + b _ { 1 } ) ^ { 2 } + ( a _ { 2 } + b _ { 2 } ) ^ { 2 } } ( b _ { 1 } ^ { 2 } + b _ { 2 } ^ { 2 } ) \left(a_{1} b_{2}\right.\right. \\
& \left.-a_{2} b_{1}\right)\left(a_{2}+b_{2}\right)\left(a_{3}+b_{3}\right)
\end{aligned}
$$

$$
\begin{aligned}
& -b_{2} b_{3}\left(a_{1} b_{2}-a_{2} b_{1}\right)-a_{1}\left(-b_{3}\left(a_{1} b_{1}+a_{2} b_{2}\right)\right. \\
& \left.\left.+a_{3}\left(b_{1}^{2}+b_{2}^{2}\right)\right)\right), \\
\delta= & \frac{1}{\left(a_{1}+b_{1}\right)^{2}+\left(a_{2}+b_{2}\right)^{2}}\left(b_{1}^{2}+b_{2}^{2}\right)\left(a_{1} b_{2}-a_{2} b_{1}\right) .
\end{aligned}
$$

This algebra has a complicated form because the bases (2.10) and (2.11) are complicated.

General independent solutions of (2.7) are given by

$v_{1}^{i}=\epsilon^{i j k} a_{j} l_{k}(a)$,

$v_{2}^{i}=\epsilon^{i j k} a_{j} m_{k}(a)$,

where $a, l(a)$, and $m(a)$ are all independent for all $a$. The corresponding generators are given by

$T_{1}(a):=\mathrm{e}^{i a \cdot x} \operatorname{det}(l a \partial)$,

$T_{2}(a):=\mathrm{e}^{i a \cdot x} \operatorname{det}(m a \partial)$,

where $\operatorname{det}(a b c):=\epsilon^{i j k} a_{i} b_{j} c_{k}$.

If we choose $l=(0,0,1)$ and $m=\left(-a_{2}, a_{1}, 0\right)$ for $a=$ $\left(a_{1}, a_{2}, a_{3}\right)$ (except $\left.a_{1}=a_{2}=0\right),(2.14)$ and (2.15) represent (2.8) and (2.10), respectively. If we choose $l=\left(0,-\frac{1}{a_{3}}, 0\right)$ and $m=\left(\frac{1}{a_{3}}, 0,0\right)$ for $a=\left(0,0, a_{3}\right),(2.14)$ and $(2.15)$ represent (2.9) and (2.11), respectively.

\section{Simple structure constants of VPD algebra}

In this section, we search for a complete independent basis that gives more simple structure constants. Although (2.15) for constant $l$ and $m$ are not independent in a part of the region of $a$ where $a$ is on a plane spanned by $l$ and $m$, we can calculate the commutation relations among (2.15) for constant $l$ and $m$, and then obtain simple relations:

$\left[T_{1}(a), T_{1}(b)\right]=i \operatorname{det}(l a b) T_{1}(a+b)$,

$\left[T_{2}(a), T_{2}(b)\right]=i \operatorname{det}(m a b) T_{2}(a+b)$,

$\left[T_{1}(a), T_{2}(b)\right]=i \frac{1}{\operatorname{det}(\operatorname{lm}(a+b))}(\operatorname{det}(m a b) \operatorname{det}(\operatorname{lma})$

$$
\left.\times T_{1}(a+b)+\operatorname{det}(l a b) \operatorname{det}(\operatorname{lmb}) T_{2}(a+b)\right) .
$$

For example, if we choose $l=(0,0,1)$ and $m=(1,0,0)$, we obtain $v_{1}=\left(-a_{2}, a_{1}, 0\right), v_{2}=\left(0,-a_{3}, a_{2}\right)$, and the corresponding generators

$U_{1}(a)=\mathrm{e}^{i a x}\left(-a_{2} \partial_{1}+a_{1} \partial_{2}\right)$,

$U_{2}(a)=\mathrm{e}^{i a x}\left(-a_{3} \partial_{2}+a_{2} \partial_{3}\right)$.

In this case, for $a_{2}=0, v_{1}=\left(0, a_{1}, 0\right)$ and $v_{2}=\left(0,-a_{3}, 0\right)$ are dependent, and thus $U_{1}(a)$ and $U_{2}(a)$ are.

Then we choose a step function, $m=(1,0,0)$ for $a_{2} \neq 0$ and $m=(0,1,0)$ for $a_{2}=0$. When $m=(0,1,0)$ we have 
$v_{3}=\left(a_{3}, 0,-a_{1}\right)$ and

$U_{3}(a)=\mathrm{e}^{i a x}\left(a_{3} \partial_{1}-a_{1} \partial_{3}\right)$,

which is independent of $U_{1}(a)$ and $U_{2}(a)$ for $a_{2}=0$. After considering $v_{1}=0$ when $a_{1}=a_{2}=0$, we have a complete set of independent generators,

$U_{1}(a)\left(\right.$ except $\left.a_{1}=a_{2}=0\right)$,

$U_{2}(a)$ (except $\left.a_{2}=0\right)$,

$U_{2}\left(0,0, a_{3}\right)$,

$U_{3}\left(a_{1}, 0, a_{3}\right)$.

In fact, for each $a$ there are two independent generators:

$U_{1}(a)$ and $U_{2}(a)$ for $a_{2} \neq 0$,

$U_{1}(a)$ and $U_{3}(a)$ for $a_{2}=0$ and $a_{1} \neq 0$,

$U_{2}(a)$ and $U_{3}(a)$ for $a_{2}=a_{1}=0$.

Then we obtain the simple structure constants of the VPD algebra,

$$
\begin{aligned}
& {\left[U_{1}(a), U_{1}(b)\right]=i\left(a_{1} b_{2}-a_{2} b_{1}\right) U_{1}(a+b)} \\
& {\left[U_{2}(a), U_{2}(b)\right]=i\left(a_{2} b_{3}-a_{3} b_{2}\right) U_{2}(a+b)} \\
& {\left[U_{3}\left(a_{1}, 0, a_{3}\right), U_{3}\left(b_{1}, 0, b_{3}\right)\right]} \\
& \quad=i\left(a_{3} b_{1}-a_{1} b_{3}\right) U_{3}\left(a_{1}+b_{1}, 0, a_{3}+b_{3}\right), \\
& \quad\left[U_{1}(a), U_{2}(b)\right]=i \frac{1}{a_{2}+b_{2}}\left(a_{2}\left(a_{2} b_{3}-a_{3} b_{2}\right) U_{1}(a+b)\right. \\
& \left.\quad+b_{2}\left(a_{1} b_{2}-a_{2} b_{1}\right) U_{2}(a+b)\right), \\
& \left.\quad \times U_{1}(a), U_{3}\left(b_{1}, 0, b_{3}\right)\right]=i\left(\left(-a_{1} b_{3}+a_{3} b_{1}+b_{1} b_{3}\right)\right. \\
& \left.\quad \times U_{1}(a+b)+b_{1}^{2} U_{2}(a+b)\right), \\
& \quad+\left(a_{3}(a), U_{3}\left(b_{1}, 0, b_{3}\right)\right]=i\left(-b_{3}^{2} U_{1}-b_{1}(a+b) U_{2}(a+b)\right) .
\end{aligned}
$$

From (3.8), (3.9), and (3.10), one can see three APD algebras corresponding to the $\left(x^{1}, x^{2}\right),\left(x^{2}, x^{3}\right)$, and $\left(x^{3}, x^{1}\right)$ planes.

\section{Conclusion and discussion}

In this paper, we found general complete independent bases of the VPD algebra. Especially, we found a complete independent basis that gives simple structure constants where the three APD algebras are manifest. This implies that an algebra that regularizes a VPD algebra should include three $u(N)$ Lie algebras.

Acknowledgments We would like to thank T. Asakawa, K. Hashimoto, N. Ikeda, N. Kamiya, H. Kunitomo, T. Matsuo, S. Moriyama, K. Murakami, J. Nishimura, S. Sasa, P. Schupp, F. Sugino, T. Tada, S. Terashima, S. Watamura, K. Yoshida, and especially H. Kawai and A. Tsuchiya for valuable discussions. This work is supported in part by a Grant-in-Aid for Young Scientists (B) No. 25800122 from JSPS.
Open Access This article is distributed under the terms of the Creative Commons Attribution License which permits any use, distribution, and reproduction in any medium, provided the original author(s) and the source are credited

Funded by $\mathrm{SCOAP}^{3}$ / License Version CC BY 4.0.

\section{References}

1. D.B. Fairlie, P. Fletcher, C.K. Zachos, Trigonometric structure constants for new infinite algebras. Phys. Lett. B 218, 203 (1989)

2. J. Hoppe, Diffeomorphism groups, quantization and SU(infinity). Int. J. Mod. Phys. A 4, 5235 (1989)

3. D.B. Fairlie, C.K. Zachos, Infinite dimensional algebras, sine brackets and SU(Infinity). Phys. Lett. B 224, 101 (1989)

4. B. de Wit, U. Marquard, H. Nicolai, Area preserving diffeomorphisms and supermembrane Lorentz invariance. Commun. Math. Phys. 128, 39 (1990)

5. T. Banks, W. Fischler, S.H. Shenker, L. Susskin, M theory as a matrix model: a conjecture. Phys. Rev. D 55, 5112-5128 (1997)

6. B. de Wit, J. Hoppe, H. Nicolai, On the quantum mechanics of supermembranes. Nucl. Phys. B 305, 545 (1988)

7. N. Ishibashi, H. Kawai, Y. Kitazawa, A. Tsuchiya, A Large N reduced model as superstring. Nucl. Phys. B 498, 467-491 (1997)

8. M. Sato, Covariant formulation of M-Theory. Int. J. Mod. Phys. A 24, 5019-5024 (2009)

9. M. Sato, Model of M-theory with eleven matrices. JHEP 1007, 026 (2010)

10. M. Sato, Supersymmetry and DLCQ Limit of Lie 3-algebra Model of M-theory. Phys. Rev. D 85, 046003 (2012)

11. M. Sato, Three-algebra BFSS matrix theory. Int. J. Mod. Phys. A 28, 1350155 (2013)

12. M. Sato, Extension of IIB matrix model by three-algebra. Int. J. Mod. Phys. A 28, 1350083 (2013)

13. M. Sato, Four-algebraic extension of the IIB matrix model. PTEP 2013, 073B04 (2013)

14. Y. Nambu, Generalized Hamiltonian dynamics. Phys. Rev. D 7, 2405-2414 (1973)

15. E.G. Floratos, The Heisenberg-weyl group on the $Z(N) \mathrm{X} Z(N)$ discretized torus membrane. Phys. Lett. B 228, 335 (1989)

16. R. Chatterjee, Dynamical symmetries and Nambu mechanics. Lett. Math. Phys. 36, 117-126 (1996)

17. J. Hoppe, On M algebras, the quantization of Nambu mechanics, and volume preserving diffeomorphisms. Helv. Phys. Acta 70, 302-317 (1997)

18. G. Dito, M. Flato, D. Sternheimer, L. Takhtajan, Deformation quantization and Nambu mechanics. Commun. Math. Phys. 183, 1-22 (1997)

19. D. Sternheimer, Deformation quantization: twenty years after. in Particles, Fields, and Gravitation, volume 453 of American Institute of Physics Conference Series, ed. by J. Rembielinski (1998), p. 107-145

20. H. Awata, M. Li, D. Minic, T. Yoneya, On the quantization of Nambu brackets. JHEP 0102, 013 (2001)

21. D. Minic, $M$ theory and deformation quantization (1999). arXiv:hep-th/9909022

22. D. Minic, Towards covariant Matrix theory (2000). arXiv:hep-th/0009131

23. C.M. Hofman, W.K. Ma, Deformations of closed strings and topological open membranes. JHEP 0106, 033 (2001)

24. T. Curtright, C.K. Zachos, Classical and quantum Nambu mechanics. Phys. Rev. D 68, 085001 (2003)

25. H. Shimada, Membrane topology and matrix regularization. Nucl. Phys. B 685, 297-320 (2004) 
26. C.K. Zachos, Membranes and consistent quantization of Nambu dynamics. Phys. Lett. B 570, 82-88 (2003)

27. A. De Castro, M.P. Garcia del Moral, I. Martin, A. Restuccia, M5brane as a Nambu-Poisson geometry of a multiD1-brane theory. Phys. Lett. B 584, 171-177 (2004)

28. M. Axenides, E. Floratos, Euler top dynamics of Nambu-Gotto p-Branes. JHEP 0703, 093 (2007)

29. C.-S. Chu, P.-M. Ho, Y. Matsuo, S. Shiba, Truncated NambuPoisson bracket and entropy formula for multiple membranes. JHEP 0808, 076 (2008)

30. M. Axenides, E. Floratos, Nambu-Lie 3-algebras on fuzzy 3manifolds. JHEP 0902, 039 (2009)

31. N. Akerblom, C. Saemann, M. Wolf, Marginal deformations and 3-algebra structures. Nucl. Phys. B 826, 456-489 (2010)

32. M. Axenides, E.G. Floratos, S. Nicolis, Nambu quantum mechanics on discrete 3-Tori. J. Phys. A 42, 275201 (2009)
33. N. Sasakura, A renormalization procedure for tensor models and scalar-tensor theories of gravity. Int. J. Mod. Phys. A 25, 4475$4492(2010)$

34. A. Gustavsson, An associative star-three-product and applications to $\mathrm{M}$ two/M five-brane theory. JHEP 1011, 043 (2010)

35. P. Bouwknegt, B. Jurco, AKSZ construction of topological open p-brane action and Nambu brackets. ArXiv e-prints (2011)

36. M. Sato, Zariski quantization as second quantization. Phys. Rev. D 85, 126012 (2012)

37. B. Jurco, P. Schupp, Nambu-Sigma model and effective membrane actions. Phys. Lett. B 713, 313-316 (2012) 